\title{
Asociación entre Hiperlaxitud Articular Generalizada y Chasquidos de la Articulación Témporo Mandibular
}

\author{
Association between General Joint Hypermobility \\ and Temporomandibular Joint Clicking \\ Associação entre Hiperlaxidade Articular Generalizada e \\ Cliques de Articulação Temporomandibular \\ Martín Sanguinetti ${ }^{1}$ (D) 0000-0002-8300-7712 \\ Martín Minvielle ${ }^{2}$ DD 0000-0002-1706-6415 \\ Raúl Riva' (iD) 0000-0002-8701-9286
}

\section{Resumen}

El objetivo del estudio fue analizar si la hiperlaxitud articular generalizada (HAG) es un factor de riesgo para los trastornos de la articulación temporomandibular (ATM). Para ello se evaluó la posible asociación entre chasquido de la ATM y el diagnóstico de HAG. Se trabajó con la siguiente hipótesis: el paciente con HAG, tendría mayor prevalencia de chasquido a nivel de la ATM que los que no la presentan, constituyendo la HAG un factor de riesgo para padecer un desorden articular (DA).

Se examinaron 214 estudiantes de facultad de odontología (FO) de la Universidad de la República (Udelar) de Uruguay, 161 participantes del sexo femenino y 53 masculino, de entre 18 y 30 ańos (edad media 23.8 años, $\mathrm{DE}=2.7$ ). A cada participante se le realizó un cuestionario y un examen clínico para diagnóstico de HAG utilizando el índice de Beighton (IB), chasquido, antecedente de trauma maxilofacial, ortodoncia, dentición completa, bloqueo abierto y turno. Todos los exámenes fueron realizados por un investigador ciego calibrado (calibración interoperador chasquido kappa $=0.68$; intraoperador Beighton=0.82, chasquido=1). El estudio fue aprobado por el Comité de Ética y todos los participantes firmaron un consentimiento informado. El análisis estadístico de los datos fue realizado en base a un modelo de regresión logística múltiple.

La prevalencia de HAG fue $34.16 \%$ en el género femenino y $7.55 \%$ en el masculino, de chasquido $24.22 \%$ para el femenino y $11.32 \%$ en el masculino. Las variables género $(\mathrm{OR}=3,244$, p-valor 0,018$)$ y antecedente de traumatismo $(\mathrm{OR}=2,478$, $\mathrm{p}$-valor 0,041$)$ se asociaron significativamente a la presencia de chasquido. No se encontró asociación entre chasquido e HAG.

El género femenino y los antecedentes de traumatismo podrían ser factores de riesgo para desórdenes a nivel de la ATM. La ausencia de asociación entre HAG y chasquido en dicho grupo etario (18-30 años), sugiere que dicho factor podría no ser de riesgo para el desarrollo de dichas patologías.

Palabras clave: Articulación Temporomandibular, Inestabilidad de la Articulación.

1 Departamento de Trastornos Témporo Mandibulares y Bruxismo, Facultad de Odontología, Universidad de la República, Uruguay. dr.sanguinetti@hotmail.com

2 Cátedra de Rehabilitación, Prostodoncia Fija y Trastornos Temporomandibulares, Facultad de Odontología de la Universidad de la República, Uruguay.

Fecha de recibido: 17/9/2020 - Fecha de aprobado: 2/2/2021 


\section{Abstract}

The objective of the study was to examine whether generalized joint hyperlaxity $(\mathrm{GJH})$ is a risk factor for temporomandibular joint disorders (TMD). This was done by assessing the possible association between temporomandibular joint (TMJ) clicks and GJH diagnosis. Work was done with the following hypothesis: the patient with GJH, would have a higher prevalence of click at the TMJ level than those who do not, making GJH a risk factor for TMJ disorders.

214 students of dental school from the Universidad de la República (Udelar) of Uruguay, 161 female and 53 male participants, aged between 18 and 30 years (average age 23.8 years, DE: 2.7) were examined. Each participant was given a questionnaire and clinical examination for HAG diagnosis using the Beighton (BI) index, click, maxillofacial trauma antecedent, orthodontics, complete teeth, open block, and shift. All tests were performed by a calibrated blind researcher (kappa click: 0.68 interoperator calibration; Beighton: 0.82 intraoperator, click: 1). The study was approved by the Ethics Committee and all participants signed informed consent. Statistical analysis of the data was performed on the basis of a multiple logistic regression model.

The prevalence of HAG was $34.16 \%$ in the female gender and $7.55 \%$ in the male, click was $24.22 \%$ for female and $11.32 \%$ male. The gender variables (OR-3, 244, p-value 0.018) and antecedent of trauma (OR-2, 478, p-value 0.041 ) were significantly associated with the presence of click. No association was found between click and GJH.

Female gender and a history of trauma could be risk factors for TMJ disorders. The absence of association between HAG and click suggests that this factor may not be a risk for the development of such pathologies.

Keywords: Temporomandibular Disorders, Joint Instability.

\section{Resumo}

O objetivo do estudo foi examinar se a hiperlaxidade articular generalizada (HAG) é um fator de risco para disfuncao articulacao temporomandibular (DAT). Isso foi feito avaliando a possível associação entre cliques atm e diagnóstico hag. $\mathrm{O}$ trabalho foi feito com a seguinte hipótese: o paciente com HAG, teria maior prevalência de clique no nível atm do que aqueles que não o fazem, tornando a HAG um fator de risco para DAT. Foram examinados 214 alunos de Odontologia da Universidad de la República (Udelar) do Uruguai, 161 do sexo feminino e 53 do sexo masculino, com idade entre 18 e 30 anos (idade média de 23, 8 anos, DE: 2, 7). Cada participante recebeu questionário e exame clínico para diagnóstico de HAG utilizando o índice Beighton (IB), clique, antecedente de trauma maxilofacial, ortodontia, dentição completa, bloqueio aberto e turno. Todos os testes foram realizados por um pesquisador cego calibrado (calibração interoperadora kappa-click-0, 68; intraoperador Beighton- 0.82 , clique- 1 ). O estudo foi aprovado pelo Comitê de Ética e todos os participantes assinaram consentimento informado. A análise estatística dos dados foi realizada com base em um modelo de regressão logística múltipla.

A prevalência de HAG foi de $34,16 \%$ no sexo feminino e $7,55 \%$ no masculino, de clique 24 , $22 \%$ para o feminino e $11,32 \%$ para o masculino. As variáveis de gênero (OR-3.244, valor $\mathrm{p} 0$, 018 ) e antecedente do trauma (OR-2.478, valor p 0,041) estiveram significativamente associadas à presença de clique. Não foi encontrada associação entre clique e HAG.

O sexo feminino e o histórico de trauma podem ser fatores de risco para distúrbios no nível do articulacao temporomandibular. A ausência de associação entre HAG e click sugere que esse fator pode não estar em risco para o desenvolvimento de tais patologias.

Palavras-chave: Articulação Temporomandibular, Inestabilidade Articular. 


\section{Introducción}

En el pasado se han definido a los Desórdenes Temporomandibulares (DTM) como un grupo heterogéneo de condiciones que afectan a la articulación temporomandibular (ATM), a los músculos masticadores (MM) y estructuras relacionadas ${ }^{(1)}$. Recientemente se los han considerado como afecciones de etiología multifactorial, donde interactúan varios sistemas (sicológico-genético-hormonal-neurológico) con factores anatómicos ${ }^{(2)}$. Dentro de los DTM, se encuentran los desórdenes articulares (DA) a nivel de la $\operatorname{ATM}^{(3)}$. Frecuentemente los DA surgen como cuadros patológicos leves, pudiendo evolucionar a cuadros de mayor severidad ${ }^{(4)}$. Las manifestaciones clínicas que se asocian a los trastornos iniciales de la ATM son ruidos del tipo chasquido y dolor ${ }^{(5)}$. En un relevamiento nacional realizado en Uruguay se reportó una prevalencia de chasquido en el examen clínico de 29, 77\% para Montevideo y 23, 01\% para el resto del país ${ }^{(6)}$. Cerca del $19 \%$ de la población sueca muestra síntomas de DA, chasquido recíproco el $7 \%$ y bloqueo cerrado crónico el $12 \%{ }^{(7)}$. El chasquido articular es considerado una variable predictora de DA y ha sido considerada su principal manifestación clínica ${ }^{(8)}$. El desplazamiento discal con reducción (DDcR) se ha asociado a aplanamiento condilar, y el desplazamiento discal sin reducción (DDsR) a cambios óseos degenerativos ${ }^{(9)}$. La prevalencia de los DTM y de los DA es superior en mujeres que en hombres ${ }^{(6,10)}$. Los factores de riesgo generales y locales podrían explicar esa diferen$\mathrm{cia}^{(8)}$. El conocimiento de los factores predisponentes y desencadenantes podrían colaborar en la prevención de los DTM, mientras que el entendimiento de los factores perpetuantes, incrementa las posibilidades de tener éxito en el tratamiento ${ }^{(11)}$.

La hiperlaxitud articular (HA) ha sido estudiada como factor de riesgo para los DA y se define como un rango aumentado de movilidad articular, en comparación con la población general, pudiendo ser observada como una condi- ción local, hiperlaxitud articular local (HAL) o general, la hiperlaxitud articular generalizada ${ }^{(12)}$ (HAG). La HAG puede ser consecuencia de un defecto del colágeno, como en los síndromes de Ehlers-Danlos o Marfan, aunque también puede presentarse sin defecto del colágeno subyacente. En dichos casos se podría hacer referencia a la HAG Benigna ${ }^{(13)}$ (HAGB).

El Índice de Beighton (IB) (Fig. 1) ha ganado aceptación internacional y parece ser el más empleado para el diagnóstico de la HAG en la literatura científica ${ }^{(14)}$.

El IB ha sido validado para ser usado por odontólogos ${ }^{(15)}$. Varios estudios han sido realizados con el objetivo de analizar la posible asociación entre DA y HAG.

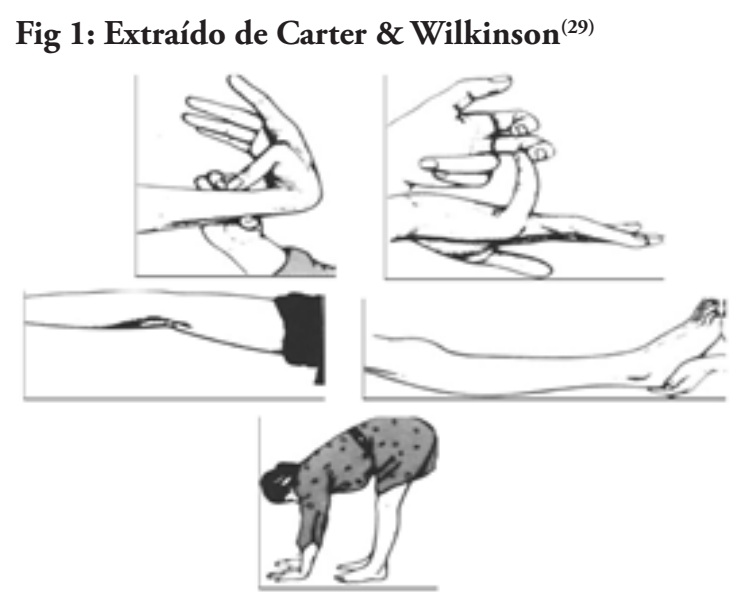

En una única revisión sistemática sobre el tema, se concluyó la existencia de una asociación, aunque no se podría aseverar que la $\mathrm{HAB}$ fuese un factor de riesgo para los $\mathrm{DA}^{(12)}$. En un estudio caso-control se encontró mayor prevalencia de HAG y DTM en la franja etaria de 15-24 y existencia de asociación entre HAG y chasquido, los autores concluyeron que el riesgo de tener DA es mayor en individuos con $\mathrm{HAG}^{(16) \text {. }}$ En un estudio en 701 pacientes jóvenes (77\% mujeres) el 63\% de los pacientes con DA fue diagnosticado con HAG, estableciéndose un elevado nivel de asociación ${ }^{(17)}$. En una muestra poblacional de 1600 individuos la prevalen- 
cia reportada de HAG fue de 6.9\%. y de DA $14.8 \%$, los autores concluyeron que la HAG se asoció a DTM tipo DDCR ${ }^{(18)}$. Ting-Han et al. realizaron un estudio retrospectivo ${ }^{(19)}$, sobre 975.788 individuos aleatoriamente seleccionados, concluyeron que la confirmación de la asociación entre DA y HAG, permite sugerir que los individuos con TTM y HAG deberían ser tratados por un equipo multidisciplinario. En un estudio reciente tipo caso-control ${ }^{(20)}$ se empleó el IB y el RDC/TMD, los autores concluyeron que el riesgo de contraer un DA es mayor en pacientes con ciertas patologías sistémicas, como la HAG entre otras.

Con resultados opuestos, Barrerara Mora et al. ${ }^{(21)}$ estudiaron 140 pacientes de ambos géneros con necesidad de tratamiento ortodóncico, reportaron una prevalencia de HAG de 28.\%, no encontrando asociación entre diferentes tipos de TTM y la HAG. En un estudio con diseño tipo caso-control que incluyó 60 casos y 60 controles $^{(22)}$ (edad media de 25 años), los autores concluyeron que la HAG no contribuye en la aparición de los TTM, ya que observaron que este es un hallazgo común en pacientes asintomáticos. Sáez-Yuguero et al. analizaron 66 mujeres jóvenes en atención por TTM, reportando un IB del 51\% y 40\% de DDCR en la muestra, concluyendo que utilizando IB y resonancia magnética para diagnóstico no se podría afirmar que la HAG sea un factor de riesgo para la $\operatorname{ATM}^{(23)}$..

En un estudio transversal y prospectivo ${ }^{(24)}$ se evaluaron 34 mujeres jóvenes con diagnóstico de DA, la prevalencia de HAG fue del 65\%, no pudiendo establecer a la HAG como factor de riesgo para TTM.

En un estudio tipo caso-control se incluyeron 42 pacientes quirúrgicos y 20 controles, encontrando 9.6 veces más chance de desarrollar chasquido recíproco con la presencia de $\mathrm{HAB}$, concluyendo que la HAG es un factor etiológico de peso para el desarrollo de chasquido recíproco y bloqueo cerrado crónico de la $\operatorname{ATM}^{(25)}$. En otro diseño de caso control ${ }^{(26)}$ también se utilizó la resonancia magnética para el diagnóstico articular, los autores concluyeron que basados en el IB, la HAG no es un factor predictor de la presencia de DA.

Los resultados de estos estudios son controversiales. Algunos estudios han encontrado asociación entre la HAGB y los DA, mientras que otros no han podido probar dicha asociación.

Los objetivos de este trabajo fueron:

- evaluar la diferencia de prevalencia entre género de HAG y chasquido.

- estudiar la posible asociación entre chasquidos articulares y la HAG.

Se trabajó con la siguiente hipótesis: el paciente con HAG, tendría mayor prevalencia de chasquido a nivel de la ATM que los que no la presentan, constituyendo la HAG un factor de riesgo para los DA.

\section{Materiales y métodos}

\section{Participantes}

Se diseñó un estudio transversal donde se relevaron estudiantes de la FO de la Udelar entre 18 y 30 años de edad. Se seleccionaron en forma consecutiva, de lunes a viernes en los dos turnos horarios de clínica, de 8: 00 a 12: 00 y de 19: 30 a 23: 30, durante los meses de octubre, noviembre, diciembre de 2017, y marzo de 2018. El protocolo del estudio fue aprobado por el comité de ética de la FO de la Udelar (Uruguay). A cada participante le fue realizado un interrogatorio y un examen clínico que incluyó el IB, en una única sesión.

\section{Tamaño muestral}

Para el cálculo del tamaño muestral se tuvieron en cuenta las prevalencias anteriormente reportadas $^{(21)}$. Considerando un nivel de $5 \%$ y una potencia de $80 \%$, el tamaño de muestra resultante fue de $200(+25$ por no respuesta), 150 para género femenino y 50 masculino de manera de poder establecer diferencias en preva- 
lencia de HAG entre género. No se registraron negativas a participar.

Los siguientes criterios fueron tenidos en cuenta para su selección:

Criterios de inclusión:

- Estudiantes de la FO de la Udelar

- Individuos de ambos géneros de entre 18 y 30 años.

- El participante estuvo de acuerdo en formar parte del estudio clínico, y lo expresó en forma escrita

Criterios de exclusión:

- Antecedentes de cirugía de ATM

- Cualquier enfermedad general que afecte el tejido conectivo o las articulaciones

- Traumatismos con secuelas de movilidad articular permanente

- Prótesis articular

- Hiperlaxitud extrema (ej. Bailarines, atletas profesionales)

\section{Variables}

A cada participante se le realizó un cuestionario y examen clínico, que incluyó el IB, en una única entrevista, siendo el operador ciego a los objetivos del estudio. El examinador es miembro de la Cátedra de Rehabilitación, Prostodoncia fija y Trastornos Temporomandibulares, con experiencia en el campo. Dicho examinador fue calibrado y realizó la medición de las variables estudiadas (interoperador chasquido kappa $=0.68$, intraoperador Beighton $=0.82$, chasquido=1). La tabla 1 muestra el resultado de las calibraciones correspondientes.

Chasquido: se siguieron los conceptos del DC/ $\mathrm{RDC}^{(3)}$ y el protocolo de calibración del estudio de Riva et al. en $2011^{(6)}$. El examinador explicó que las ATM serían examinadas para determinar si había presencia de ruidos articulares durante apertura y cierre. Localizando los dedos índice y mayor sobre la piel que cubre los polos laterales de ambas ATM simultáneamente mientras el participante realizaba 3 movimientos de apertura máxima y cierre, comenzando
Tabla 1. Calibración de medidas

\begin{tabular}{|l|c|}
\hline \begin{tabular}{l|} 
variable \\
inter
\end{tabular} & kappa \\
\hline chasquido & 0.679 \\
\hline beighton 1 & 0.800 \\
\hline beighton 2 & 0.824 \\
\hline beighton 3 & 0.800 \\
\hline beighton 4 & 0.800 \\
\hline beighton 5 & 0.750 \\
\hline beighton 6 & 1.000 \\
\hline beighton 7 & - \\
\hline beighton 8 & - \\
\hline beighton 9 & 0.800 \\
\hline intra & \\
\hline chasquido & 1.000 \\
\hline
\end{tabular}

y terminando dichos movimientos en posición de máxima intercuspidación. Cuando el ruido fue reproducido en 1 de 3 ciclos, en una o ambas ATM, tanto únicamente en apertura, así como en apertura y cierre, el participante fue diagnóstico positivo para ruido articular tipo chasquido.

Se realizó el diagnóstico diferencial, en relación a las alteraciones de forma, descartando ruidos articulares recíprocos en el mismo rango en apertura y cierre, siendo por lo tanto chasquido negativo.

La presencia de la dentición total (DentT) y la ausencia de molares 6, 7 y 8 (Molares) del mismo cuadrante fueron evaluadas mediante un examen clínico intrabucal con instrumental de inspección, espejo, pinza, sonda, periodontímetro y luz de equipo odontológico.

Dent T fue positiva cuando se encontraron presentes todos los incisivos, premolares y molares, contemplando la ausencia o presencia de alguno o todos los terceros molares.

Molares fue positiva cuando se constató la ausencia clínica de primer, segundo y tercer molar en un mismo cuadrante.

Bloqueo abierto e historia de traumatismo fueron positivas, cuando el participante lo relató en la anamnesis 
El índice de Beighton se realizó de la manera propuesta por dicho autor, y ampliada en su descripción oral por Juul-Kristensen et al. en $2007^{(14)}$, utilizando para ello un goniómetro.

\section{Índice de Beighton}

1.Dorsiflexión pasiva del pulgar, teniendo la muñeca en flexión

2.Dorsiflexión pasiva del dedo meñique a más de $90^{\circ}$ con muñeca extendida

3. Hiperextensión activa de los codos 10 grados o más

4.Hiperextensión activa de las rodillas 10 grados o más

5.Flexión del tronco hacia delante tocando el suelo con las palmas de las manos al agacharse sin doblar las rodillas

El rango de puntuación fue de 0-9, las mayores puntuaciones denotaron mayor hiperlaxitud. Se consideraron Beighton positivo aquellos participantes con 4 o más puntos. No se categorizaron a los pacientes en función de las articulaciones hipermóviles.

\section{Análisis estadístico}

En primer lugar se realizó un análisis descriptivo de las variables considerando distribución de frecuencias para las variables cualitativas. Mientras que para las cuantitativas se calculó media y desvío estándar. En segundo lugar se consideró un análisis bivariado entre chasquido y los posibles factores de riesgo. Por último, dichos factores fueron evaluados a través de un análisis por regresión logística múltiple (RLM) y se calcularon los Odd Ratio (OR) e intervalos respectivos, teniendo en cuenta un nivel de confianza del $95 \%$.

\section{Resultados}

Se incluyeron un total de 214 participantes cuya edad promedio fue $23.8(\mathrm{DE}=2.7)$, de los cuales 161 fueron de sexo de femenino y 53 masculino. La prevalencia total de HAG fue del $27,6 \%$, de chasquido $21,0 \%$ y de historia de traumatismo $15,0 \%$. La distribución de frecuencia de las demás variables se presentan en la tabla 2 .

Tabla 2

\begin{tabular}{|l|c|c|}
\hline \multicolumn{2}{|l|}{ Frecuencia } & $\%$ \\
\hline $\begin{array}{l}\text { Género } \\
\text { masculino }\end{array}$ & 53 & $24,8 \%$ \\
\hline \begin{tabular}{l} 
femenino \\
\hline Historia de traumatismo
\end{tabular} & 161 & $75,2 \%$ \\
\hline si & 32 & $15,0 \%$ \\
\hline no & 182 & $85,0 \%$ \\
\hline Bloqueo abierto & & \\
\hline si & 4 & $1,9 \%$ \\
\hline no & 210 & $98,1 \%$ \\
\hline Ortodoncia & & \\
\hline si & 144 & $67,3 \%$ \\
\hline no & 70 & $32,7 \%$ \\
\hline Chasquido & & \\
\hline si & 45 & $21,0 \%$ \\
\hline no & 169 & $79,0 \%$ \\
\hline Dentición completa & & \\
\hline si & 7 & $3,3 \%$ \\
\hline no & 207 & $96,7 \%$ \\
\hline Hipermovilidad & & \\
\hline si & 59 & $27,6 \%$ \\
\hline no & 155 & $72,4 \%$ \\
\hline Turno & & \\
\hline matutino & 140 & $65,4 \%$ \\
\hline nocturno & 74 & $34,6 \%$ \\
\hline
\end{tabular}

La prevalencia de HAG por sexo fue mayor en el femenino siendo 34.16\% (DE 3.74\%) y 7.55\% (DE 3.66\%) para el masculino (Fig. 1). La prevalencia de chasquido fue superior también en el femenino 24.22\% (DE 3.39\%) y $11.32 \%$ (DE 4.39\%) para el sexo masculino (Gráf. 1). 


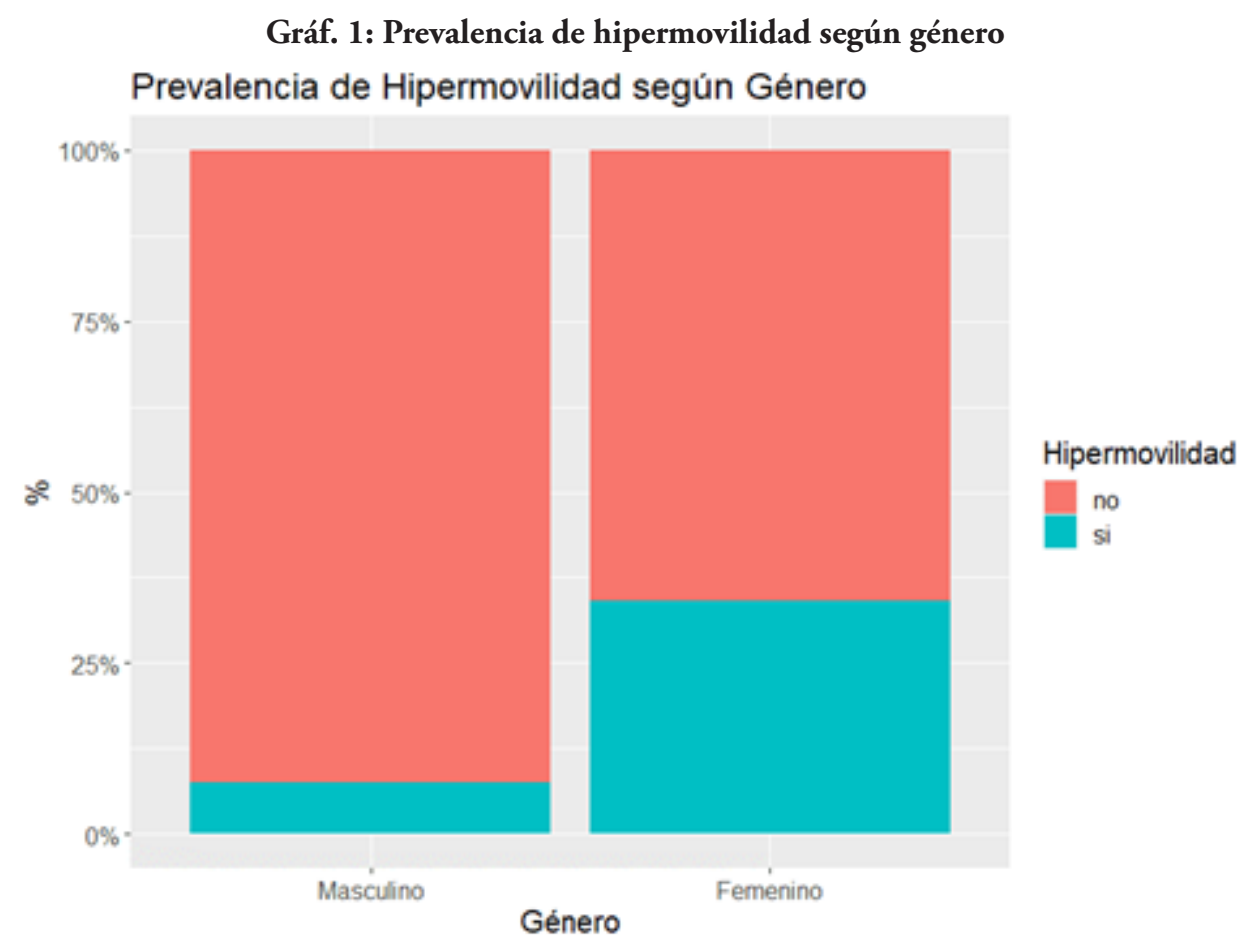

Los resultados de un primer análisis bivariado se muestran en la tabla 3.

Tabla 3

\begin{tabular}{|l|c|c|c|}
\hline \multicolumn{1}{|l|}{ Análisis bi-variado } & & & \\
\hline & chasquido no & chasquido si & p-valor \\
\hline Sexo & & & \\
\hline masculino & 88,7 & 11,3 & 0,071 \\
\hline femenino & 75,8 & 24,2 & \\
\hline Historia de traumatismo & & \\
\hline si & 81,3 & 18,7 & 0,076 \\
\hline no & 65,5 & 34,5 & \\
\hline Ortodoncia & & & \\
\hline si & 77,1 & 22,9 & 0,780 \\
\hline no & 77,9 & 22,1 & \\
\hline Hipermovilidad & & & \\
\hline si & 78,7 & 21,3 & 0,999 \\
\hline no & 79,7 & 20,3 & \\
\hline Turno & & & \\
\hline matutino & 82,1 & 17,9 & 0,165 \\
\hline nocturno & 73,0 & 27,0 & \\
\hline
\end{tabular}

Las variables, molares, Dent $\mathrm{T}$ y bloqueo no se incluyeron en el análisis bivariado ni en el modelo de regresión logística debido a que no presentaron variabilidad suficiente para evaluar asociación con la variable de respuesta. Las variables independientes, utilizadas para chasquido fueron: edad, género, historia de trauma, ortodoncia, turno e Índice de Beighton.

La prevalencia de chasquido en los participantes con y sin HAG resultó similar, siendo $21.3 \%$ y 20.3\% respectivamente (Gráf. 2). 


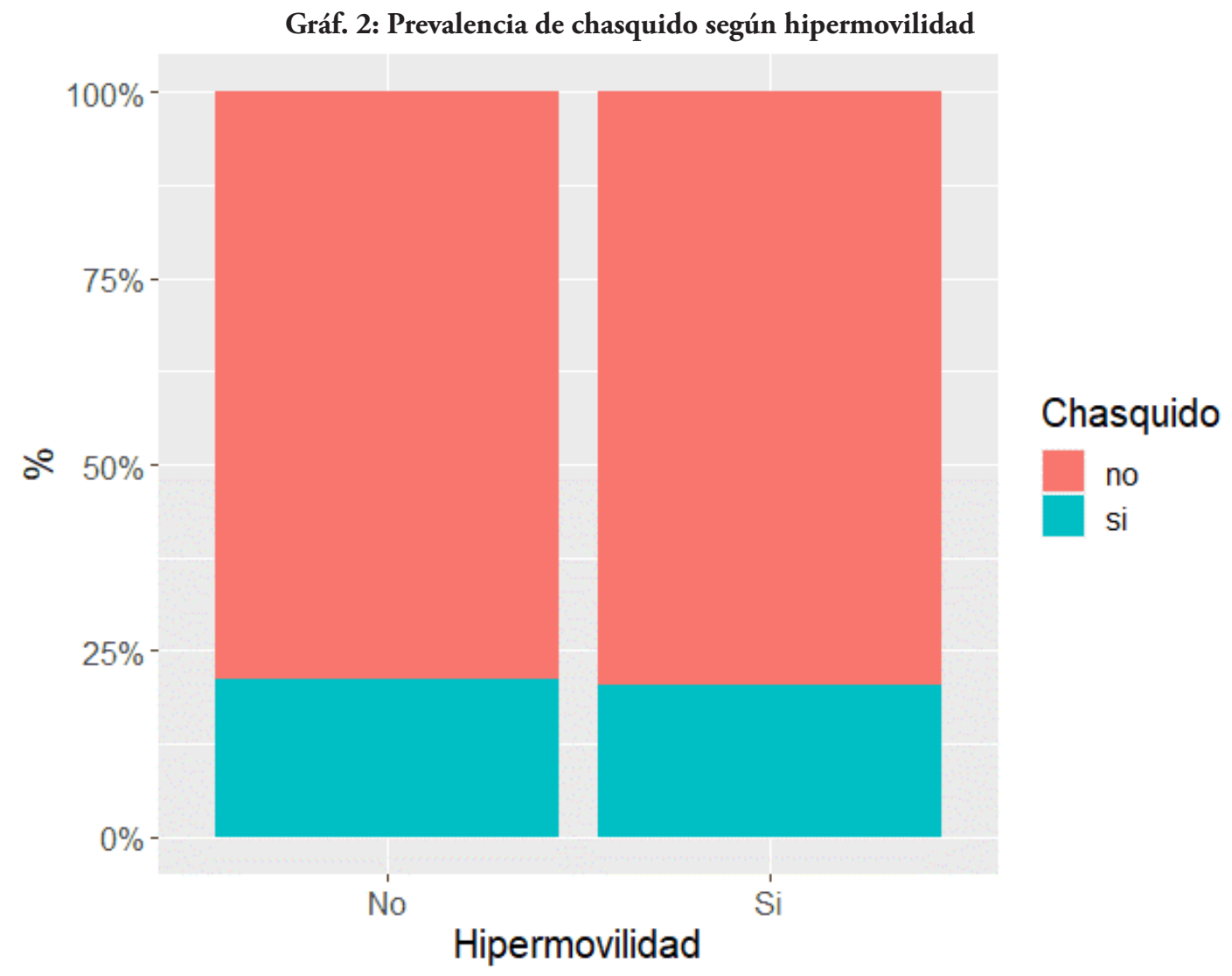

Los resultados obtenidos de cada componente del IB se describen en la tabla 4

Tabla 4

Prevalencia de cada componente del índice Beighton según género

\begin{tabular}{|c|c|c|c|c|c|c|c|c|c|c|}
\hline & $\mathrm{n}$ & Codo der. & Codo izq. & Pulgar der. & Pulgar izq. & Meñique der. & Meñique izq. & Rodilla der. & Rodilla izq. & Flexión \\
\hline Masculino & 53 & $5,7 \%$ & $5,7 \%$ & $17,0 \%$ & $26,4 \%$ & $17,0 \%$ & $22,6 \%$ & $0,0 \%$ & $1,9 \%$ & $7,6 \%$ \\
\hline Femenino & 161 & $20,5 \%$ & $26,1 \%$ & $45,3 \%$ & $47,2 \%$ & $34,8 \%$ & $39,8 \%$ & $10,6 \%$ & $11,2 \%$ & $27,3 \%$ \\
\hline Total & 214 & $16,8 \%$ & $21,0 \%$ & $38,3 \%$ & $42,1 \%$ & $30,4 \%$ & $35,5 \%$ & $7,9 \%$ & $8,9 \%$ & $22,4 \%$ \\
\hline
\end{tabular}

Tabla 5

\begin{tabular}{|c|c|c|c|c|}
\hline \multicolumn{5}{|c|}{ Regresión logística para factores de riesgo vs chasquido } \\
\hline & Estimación & $\mathbf{O R}$ & p-valor & IC $95 \%$ \\
\hline Sexo & 1,177 & 3,244 & 0,018 & $(1,298-9,422)$ \\
\hline Edad & $-0,004$ & 0,996 & 0,949 & $(0,872-1,134)$ \\
\hline Traumatismo & 0,907 & 2,478 & 0,041 & $(1,016-5,871)$ \\
\hline Ortodoncia & $-0,378$ & 0,685 & 0,308 & $(0,333-1,433)$ \\
\hline Hipermovilidad & $-0,331$ & 0,718 & 0,408 & $(0,318-1,542)$ \\
\hline Turno & 0,606 & 1,833 & 0,093 & $(0,899-3,721)$ \\
\hline
\end{tabular}


El modelo planteado intentó medir la posible asociación entre los posibles factores de riesgo y el chasquido articular como signo de desorden articular. Los resultados de la RLM se muestran en la tabla 5. El sexo femenino fue asociado como posible factor de riesgo para DA con un OR de 3,2 (p-valor 0, 018). El antecedente de traumatismo maxilofacial fue significativamente asociado a chasquido con un OR 2, 5 (pvalor 0, 041).

La edad, estar o haber estado en tratamiento ortodóncico, y el turno no fueron asociados con la prevalencia de chasquido para nuestro grupo de participantes. La HAG no se asoció significativamente al signo clínico chasquido ( $p$-valor: 0, 408 IC95\% $(0,318-1,542))$

\section{Discusión}

Los estudios publicados previamente emplearon en general diversos diseños metodológicos, dificultando en consecuencia la posibilidad de un análisis comparativo. La asociación entre DTM y HAG ha sido reportada en varios estudios, sin embargo, los resultados no son determinantes. Algunos autores han probado que la asociación existe, mientras que otros no lo han podido demostrar.

Los estudios que no revelaron asociación en línea con nuestros resultados, son en su totalidad de análisis estadístico bi-variado, estableciendo por su metodología limitaciones de inferencia estadística $^{(22,23,24,25,26)}$. La creación de un modelo de RLM permitió controlar los posibles factores de riesgo para chasquido más comunmente relatados en la literatura. En contraposición, dos estudios de diseño prospectivo en muestras de población general concluyeron que la HAG es un factor de riesgo para los DTM ${ }^{(15,20)}$.

Si bien el IB ha sido descrito como el más aceptado en la literatura ${ }^{(27)}$, otras pruebas han sido utilizadas, los criterios de Carter y Wilkinson, Rocabado Temporomandibular Pain Analysis para TTM, criterios de Brighton, lo cual podría complejizar la comparación de sus resultados con los nuestros. Así mismo dentro de los estudios relevados que utilizaron el IB, la puntuación para determinar la HAG mostró variabilidades situándose en un rango de 3 a 6 puntos positivos. Deodato et al. ${ }^{(17)}$, fijando el corte en 3 obtuvieron una prevalencia de HAG del 66\%, mientras que Pasinato et al. ${ }^{(24)}$ y Sáez Yuguero et al. ${ }^{(23)}$ utilizaron corte en 4 obteniendo prevalencias de $51 \%$ y $65 \%$ respectivamente, lo cual podría traducirse en una validez criticable del IB como elemento diagnóstico. Las prevalencias de HAG en muestras de pacientes varían entre $4 \%{ }^{(26)}$ (Wang et al., 2012) y $65 \%{ }^{(23,17)}$.

En relación a la edad, nuestra muestra fue de rango etario reducido (18-30) por lo que la variabilidad por edad no pudo ser estudiada en contraste con otros estudios de mayor rango etario en muestra de no pacientes ${ }^{(18)}$. Se ha reportado una prevalencia de HAG del $43 \%$ en adolescentes sin $\mathrm{DTM}^{(28)}$. Nuestros resultados en prevalencia de chasquidos son similares a los reportados en un relevamiento nacional ${ }^{(6)}$, y también a los estudios con muestras de población general ${ }^{(18)}$. Nuestro estudio pudo establecer diferencia por género en la prevalencia de la HAG, sin embargo, la mayoría de los estudios han sido realizados exclusivamente en mujeres $^{(24,26,28)}$, reportando otros diversas proporciones que alcanzan hasta 5: 1 a favor de las $\operatorname{mismas}^{(17),}$ sin reportes en su mayoría de prevalencias por género. En población general, sin discriminar por edad Hirsch et al. ${ }^{(18)}$ reportaron prevalencia de HAG del 6, 9\%, inferiores a las obtenidas en nuestro estudio, pero con diferencias por género.

Un solo estudio ${ }^{(25)}$ incorporó a la historia de traumatismo como variable, en un análisis bivariado, con resultado negativo de asociación, en nuestros resultados dicha variable de control demostró incidir en la prevalencia de chasquido articular (p-valor: 0,041 IC95\% (1,016 $-5,871))$. 
Ninguno de los trabajos relevados incorporó la diversidad racial en sus muestras, encontrándose los que focalizan en poblaciones europeo descendientes $^{(12,17,18,21,22,23,24,25,28)}$ y los que focalizan en poblaciones asiáticas ${ }^{(16,19,26)}$, impidiendo esto generalizar resultados.

La calibración intra e inter examinador para ambos métodos diagnósticos fue descrita sólo en tres estudios ${ }^{(18,20,26)}$. La calibración es un procedimiento necesario para otorgar rigurosidad metodológica que garantice la confiabilidad de los resultados.

\section{Conclusiones}

La hiperlaxitud articular generalizada diagnosticada por el IB fue igualmente prevalente en los participantes con y sin signos de desórdenes articulares. Los resultados del estudio sugieren que la HAG no debería considerarse un factor de riesgo para los desórdenes a nivel de la ATM en población joven (18 a 30 años). El género femenino y el antecedente de traumatismo maxilofacial podrían ser consideradas factores de riesgo para el desarrollo de patologías a nivel de la ATM.

\section{Referencias}

1. McNeill C. Introduction. En: Temporomandibular Disorders: Guidelines for classification, assessment, and managment. Chicago: Quintessense, 1993: p. 11-13.

2. Manfredini D, Poggio C. Prosthodontic planning in patients with temporomandibular disorders and/ or bruxism: a systematic review. J Prosthet Dent. 2017; 117(5): 606-613.

3. Schiffman E, Orbach R, Truelove E, Look J, Anderson G, Goulet J, List T, Svensson P, González Y. Diagnostic Criteria for Temporomandibular Disorders (DC/TMD) for Clinical and Research Applications: Recommendations of the International RDC/TMD Consortium Network and Orofacial Pain Special Interest Group. J Oral Facial Pain Headache. 2014; 28(1): 6-27.

4. Huddleston S, Lobbezoo F, Onland-Moret C, Naeije M. Anterior Disc Displacement with Reduction and Symptomatic Hypermobility in the Human Temporomandibular Joint: Prevalence Rates and Risk Factors in Children and Teenagers. J Orofac Pain. 2007; 21(1): 55-62.

5. Okeson JP. Joint Intracapsular Disorders: Diagnostic and Nonsurgical Management Considerations. Dent Clin N Am. 2007; 51 85-103.

6. Riva R, Sanguinetti M, Rodríguez A, Guzzetti L, Lorenzo S, Álvarez R, Massa F. Prevalencia de trastornos témporo mandibulares y bruxismo en Uruguay. Parte I. Odontoestomatología. 2011; 17(13): 54-71.

7. Lundh H, Westesson PL. Clinical signs of temporomandibular joint internal derangement in adults. An epidemiologic study. Oral Surg Oral Med Oral Pathol. 1991; 72(6): 637-41.

8. Magnusson T, Egermark I, Carlsson G. A prospective investigation over two decades on signs and symptoms of temporomandibular disorders and associated variables. A final summary. Acta Odontol Scand. 2005; 63: 99-109.

9. Maddalena Dias I, Rocha Coelho P, Maria Souza N, Assis P, Pessoa F, Leite P, Lopes Devito K. Evaluation of the correlation between disc displacements and degenerative bone changes of the temporomandibular joint by means of magnetic resonance images. Int. J. Oral Maxillofac. Surg. 2012; 41: 1051-1057.

10. Bueno C, Pereira D, Pattussi M, Grossi P, Grossi M. Gender differences in temporomandibular disorders in adult populational studies: a systematic review and meta-analysis. J Oral Rehabil. 2018; 45(9): 720-729.

11. Marklund S, Wanman A. Risk factors associated with incidence and persistence of signs and symptoms of temporomandibular disorders. Acta Odontol Scand. 2010; 68: 289-299. 
12. Dijkstra PU, Kropmans JB, Stegenga B. The Association between Generalized Joint Hypermobility and Temporomandibular Joint Disorders: A Systematic Review. J Dent Res 2002; 81(3): 158-163.

13. Beighton, P., Grahame, R., \& Bird, H. Assesment of hypermobility. En: Hypermobility of Joints. Inglaterra: Springer-Verlag, 1999. p9-20.

14. Juul-Kristensen B, Røgind H, Jensen DV, Remvig L. Inter-examiner reproducibility of tests and criteria for generalized joint hypermobility and benign joint hypermobility syndrome. Rheumatology. 2007; 46: $1835-1841$.

15. Hirsch C, Hirsch M., John M., Bock J.J. Reliability of the Beighton Hypermobility Index to Determinate the General Joint Laxity Performed by Dentists. J Orofac Orthop. 2007; 68: 342-52

16. Kavuncu V, Sezai S, Ayhan K, Ayse K, Cihan A. The role of systemic hypermobility and condylar hypermobility in temporomandibular joint dysfunction syndrome. Rheumatol Int. 2006; 26(3): 257-260.

17. Deodato F, Trusendi T, Giorgetti R, Scalese M. Predisposition for Temporomandibular Joint Disorders: Loose Ligaments. Cranio 2004; 24(3): 179-183.

18. Hirsch C, John MT, Stang A. Association between generalized joint hypermobility and signs and diagnoses of temporomandibular disorders. Eur J Oral Sci. 2008 ; 116(6): 525-30.

19. Ting-Han C, Da-Yo Y, Yung-Tsan W, Wan-Chien C, Fu-Gong L, Yi-Shing S, Earl F, Ren-Yeong H. The association between temporomandibular disorders and joint hypermobility syndrome: a nationwide population-based study. Clin Oral Investig. 2015; 19(8): 2123-32.

20. Fredricson A, Khodabandehlou F, Weiner C, Naimi-Akbar A, Adami J, Rosén A. Are there early signs that predict development of temporomandibular joint disease?. J Oral Sci. 2018; 60(2): 194-200.

21. Barrera-Mora JM, Espinar Escalona E, Abalos Labruzzi C, Llamas Carrera JM, Ballesteros EJ, Solano Reina E, Rocabado M. The relationship between malocclusion, benign joint hypermobility syndrome, condylar position and TMD symptoms. Cranio. 2012; 30(2): 121-30.

22. Conti PC, Miranda JE, Araujo C.R. Relationship between systemic joint laxity, TMJ hypertranslation, and intra-articular disorders. Cranio. 2000; 18(3), 192-7.

23. Sáez-Yuguero M, Linares-Tovar E, Calvo-Guirado JL, Bermejo-Fenoll A, Rodríguez-Lozano FJ. Joint hypermobility and disk displacement confirmed by magnetic resonance imaging: a study of women with temporomandibular disorders. Oral Surg Oral Med Oral Pathol Oral Radiol Endod. 2009; 107(6): 54-57.

24. Pasinato F, Souza JA, Corrêa EC, Silva AM. Temporomandibular disorder and generalized joint hypermobility: application of diagnostic criteria. Braz J Otorhinolaryngol. 2011; 77(4): 418-25.

25. Ögren M, Fältmars C, Lund B, Holmlund A. Hypermobility and trauma as etiologic factors in patients with disc derangements of the temporomandibular joint. Int J Oral Maxillofac Surg. $2012 ; 41(9)$ : 1046-50.

26. Wang HY, Shih TT, Wang JS, Shiau YY, Chen YJ. Temporomandibular joint structural derangement and general joint hypermobility. J Orofac Pain. 2012; 26(1): 33-8.

27. Remvig L, Jensen D, Ward R. Are Diagnostic Criteria for General Joint Hypermobility and Benign Joint Hypermobility Syndrome Based on Reproducible and Valid Tests? A Review of the Literature. Rheumatol 2007; 34: 798-803.

28. Winocur E, Gavish A, Halachmi M, Bloom A, Gazit E. Generalized joint laxity and its relation with oral habits and temporomandibular disorders in adolescent girls. J Oral Rehabil. 2000; 27(7): 614622.

29. Carter C, Wilkinson J. Persistent Joint Laxity and congenital Dislocation of the hip. J Bone Joint Surg Br. 1964; 46: 40-45. 


\section{Nota contribución de los autores:}

1. Concepción y diseño del estudio

2. Adquisición de datos

3. Análisis de datos

4. Discusión de los resultados

5. Redacción del manuscrito

6. Aprobación de la versión final del manuscrito

MS ha contribuido en: 1, 3, 4, 5 y 6 .

MM ha contribuido en: 2 y 6 .

RR ha contribuido en: $1,4,5$ y 6 .

Nota de aceptación:

Este artículo fue aprobado por la editora de la revista Mag. Dra. Vanesa Pereira-Prado. 